\title{
Ações de atenção primária dirigidas às mulheres de 45 a 60 anos de idade
}

\author{
Primary health care actions aimed at women aged 45 to 60 years old
}

Acciones de atención primaria dirigidas a las mujeres de 45 a 60 años de edad

Natalie Klann Garcia ${ }^{1}$, Roselane Gonçalves ${ }^{2}$, Jacqueline Isaac Machado Brigagão ${ }^{3}$

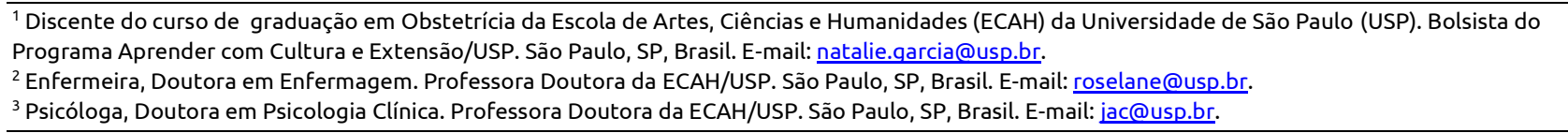

\section{RESUMO}

Estudo descritivo e exploratório que teve como objetivo identificar as ações de cuidado em saúde dirigidas às mulheres na faixa etária dos 45 aos 60 anos em uma unidade de saúde da família (USF). A coleta de dados ocorreu entre os meses de janeiro a agosto de 2011, tendo sido realizadas 16 entrevistas com os profissionais da equipe. Os resultados indicam que essa população é atendida basicamente quando busca a unidade espontaneamente. Os/as profissionais reconhecem que seria importante organizar ações de promoção da saúde, porém nem sempre é possível em virtude da quantidade de atividades diárias. Conclui-se que na unidade não há ações específicas dirigidas a essa população. O foco programático na USF está nas prioridades estabelecidas pela Estratégia de Saúde da Família (ESF), que são os hipertensos, os diabéticos, as gestantes, as crianças e os idosos.

Descritores: Cuidados de Enfermagem; Climatério; Saúde da Família; Pessoal de Saúde; Promoção da Saúde.

\section{ABSTRACT}

The objective of this descriptive and exploratory study was to identify the health care actions aimed at women within the age group of 45 to 60 years in a family health unit (FHU). Data collection took place between January and August of 2011, when 16 interviews were performed with workers of the health team. Results show that this population is basically seen when arriving at the unit spontaneously. The other workers recognize it would be important to organize health promotion actions, but that is not always possible due to the number of daily activities. In conclusion, the unit does not offer any specific actions aimed at this population. The FHU focuses its program on the priorities established by the Family Health Strategy (FHS), which are the hypertensives, diabetics, pregnant women, children and the elderly. Descriptors: Nursing Care; Climacteric; Family Health; Health Personnel; Health Promotion.

\section{RESUMEN}

Estudio descriptivo, exploratorio, que tuvo como objetivo identificar las acciones de cuidado en salud dirigidas a las mujeres en la faja etaria de 45 a 60 años en una unidad de salud de la familia (USF). Datos recolectados entre enero y agosto de 2011, habiendo sido realizadas 16 entrevistas con los profesionales del equipo. Los resultados indican que dicha población es atendida básicamente cuando se acerca a la unidad en forma espontánea. Los/las profesionales reconocen que sería importante organizar acciones de promoción de salud, aunque no siempre es posible en razón de la cantidad de actividades diarias. Se concluye en que en la unidad no hay acciones específicas dirigidas a esa población. El foco programático en la USF está en las prioridades establecidas por la Estrategia de Salud de la Familia (ESF), que son los hipertensos, los diabéticos, las embarazadas, los niños y los ancianos.

Descriptores: Atención de Enfermería; Climaterio; Salud de la Familia; Personal de Salud; Promoción de la Salud. 


\section{INTRODUÇÃO}

As ações dirigidas à saúde da mulher tem sido objeto de discussão e de elaboração de políticas públicas ao longo do tempo. Muitos esforços foram realizados para superar a tendência de focalizar as políticas nos aspectos reprodutivos e materno-infantil que, historicamente, eram os principais objetivos das políticas dirigidas às mulheres $^{(1)}$.

Nesse sentido, o Programa de Assistência Integral à Saúde da Mulher (PAISM) publicado em 1983, preconizava a integralidade da assistência como estratégia central das ações dirigidas às mulheres. Esse programa trouxe inovação no que se refere à noção ampliada sobre saúde, contrapondo-se aos programas implementados de forma verticalizada e fragmentada. Segundo as premissas do PAISM o termo "integral" refere-se ao atendimento de todas as necessidades de saúde desse grupo nas diferentes etapas do curso da vida, o que inclui as mulheres que estão vivenciando o climatério/menopausa(2-3).

No que se refere à atenção à saúde da mulher acima dos 50 anos, em 1999, a Área Técnica do Ministério da Saúde, passou a incorporar no seu planejamento estratégias de cuidado e promoção de saúde desta população(4-5). Em 2004 o documento intitulado Política Nacional de Atenção Integral à Saúde da Mulher-Princípios e Diretrizes, incluiu um capítulo sobre a atenção à saúde da mulher no climatério e menopausa instituindo assim diretrizes nacionais para o cuidado à saúde das mulheres nesta faixa etária(6).

Em São Paulo, ainda em 2003, foi criado pela Secretaria Municipal de Saúde (SMS) o Programa de Atendimento Integral e Humanizado às Mulheres em Estado de Climatério ou Pós-climatério, decretado e promulgado pela lei municipal $n^{\circ} 13.536$ e implementado em algumas unidades básicas de saúde. Os principais objetivos desse projeto eram assegurar a integralidade no cuidado à mulher nesse período da vida, diminuir a morbimortalidade dessa faixa etária e disponibilizar tratamentos ${ }^{(7)}$.

Nesse contexto, é fundamental estudar de que modo as diretrizes desses programas e políticas tem sido incorporadas nas ações desenvolvidas pela Estratégia da Saúde da Família (ESF) que centraliza as ações de Atenção Primaria a Saúde (APS) ${ }^{(8)}$.

Usualmente os termos "menopausa" e "climatério" são usados como sinônimos, mas essas duas terminologias têm significados diferentes. Fisiologicamente o climatério corresponde ao período que antecede a menopausa e se inicia por volta dos 45 anos, sendo caracterizado na literatura pelo conjunto de alterações físicas e psicológicas que acompanham a diminuição gradativa da produção hormonal, principalmente do estrogênio. Já a menopausa acontece aproximadamente em torno dos 50 anos e corresponde à cessação permanente da menstruação após 12 meses de amenorreia(9). A compreensão das mudanças e transformações vivenciadas pelas mulheres nesta fase, podem ser facilitadas por ações de promoção e educação em saúde ${ }^{(10)}$.

Assim, esta pesquisa teve como objetivo estudar as ações de cuidado em saúde dirigidas às mulheres na faixa etária dos 45 aos 60 anos, desenvolvidas por profissionais de saúde de uma Unidade de Saúde da família (USF).

\section{METODOLOGIA}

Estudo descritivo e exploratório, realizado em uma USF situada na Zona Leste de São Paulo, no período de janeiro a agosto de 2011. Essa unidade possuía duas equipes de saúde da família, denominadas também de equipes de atenção básica, compostas por dois médicos, duas enfermeiras, quatro auxiliares de enfermagem, onze agentes comunitários de saúde e uma equipe multidisciplinar, que integra o Núcleo de Apoio à Saúde da Família (NASF). A unidade abrange uma população estimada em 8.000 habitantes.

Foi incluído no estudo, no mínimo um profissional de cada categoria que atuava nas equipes de ESF. Na época da pesquisa, todos os profissionais que atuavam na unidade foram convidados a participar; 16 aceitaram e foram incluídos no estudo: duas enfermeiras, uma psicóloga, uma nutricionista, uma fonoaudióloga, um médico, seis agentes comunitárias de saúde (ACS) e quatro auxiliares de enfermagem. Foram realizadas entrevistas semiestruturadas, cujo roteiro de questões abordou as ações de saúde e a organização do cuidado prestado a essas mulheres. As entrevistas foram gravadas e os discursos transcritos na íntegra.

A partir da transcrição das entrevistas e da leitura atenta dos relatos o material foi submetido à análise qualitativa. Foram identificados quatro temas centrais: ausência de diretrizes especificas para as mulheres dos 45 aos 60 anos; demandas das mulheres da faixa etária dos 45 aos 60 anos; ações de promoção da saúde para as mulheres 
dos 45 aos 60 anos; agente comunitário como facilitador. Em cada tema foram apresentados recortes dos relatos dos profissionais, que ilustram os argumentos apresentados. A análise e a discussão foram feitas a partir das diretrizes propostas pelo Ministério da Saúde para a área da saúde da mulher no climatério e da literatura disponível sobre a temática.

Os resultados do estudo descrevem a assistência proposta e implementada no âmbito da unidade de saúde pesquisada, bem como a forma como os entrevistados percebem o cuidado que oferecem à população em questão.

A fim de manter o anonimato dos sujeitos, as falas foram identificadas com a inicial da profissão. Para as categorias com mais de um sujeito entrevistado a identificação será seguida da numeração correspondente. E1 (Enfermeiro - 1); E2 (Enfermeiro - 2); M (médico); P (Psicólogo); N (Nutricionista); F (Fonoaudióloga); ACS1 (Agente Comunitário de Saúde - 1); ACS2 (Agente Comunitário de Saúde - 2) ACS3 (Agente Comunitário de Saúde - 3); ACS4 (Agente Comunitário de Saúde - 4); ACS5 (Agente Comunitário de Saúde - 5); ACS6 (Agente Comunitário de Saúde - 6); AE1 (Auxiliar de Enfermagem - 1); AE2 (Auxiliar de Enfermagem - 2); AE3 (Auxiliar de Enfermagem - 3); AE4 (Auxiliar de Enfermagem - 4).

A pesquisa seguiu o estabelecido pela Portaria 196/1996, que trata dos estudos envolvendo seres humanos; o projeto foi avaliado e autorizado pelo Comitê de Ética em Pesquisa (CEP) da Secretaria Municipal de Saúde de São Paulo (Parecer n 336/08 - CEP/SMS). A participação dos profissionais foi voluntária e precedida de leitura, concordância e assinatura do Termo de Consentimento Livre e Esclarecido (TCLE).

\section{RESULTADOS E DISCUSSÃO}

Os resultados da pesquisa serão apresentados seguindo os quatro temas identificados nas análises: ausência de diretrizes especificas para as mulheres dos 45 aos 60 anos; demandas das mulheres da faixa etária dos 45 aos 60 anos; ações de promoção da saúde para as mulheres dos 45 aos 60 anos; agente comunitário como facilitador.

\section{Ausência de diretrizes específicas para as mulheres} dos 45 aos 60 anos

Nas entrevistas ficou evidente que o atendimento dessa população ocorria de acordo com a demanda espontânea: as consultas eram marcadas no balcão da unidade e não havia fluxo específico рага agendamento/atendimento a esse grupo. Os profissionais foram unânimes em afirmar que não há programas de saúde específicos para as mulheres dessa faixa etária, seguindo exclusivamente as prioridades estabelecidas pelas diretrizes da ESF, que preveem o cuidado aos hipertensos, aos diabéticos, às gestantes, às crianças e aos idosos, como pode ser observado na fala que se segue:

[...] elas vêm como saúde da mulher... não é prioridade pelo PSF [...] (M)

[...] se ela é hipertensa, independente da queixa... ela é encaixada na consulta de hipertensa e é visto as outras queixas dela nesse momento, porque, pra nós, a prioridade para agendamento é essa, não que a queixa dela não seja prioridade [...] (E1)

Segundo a Norma Operacional de Assistência à Saúde, 2002, a ESF fica responsável por garantir responsabilidades e ações mínimas que incluem: controle da tuberculose, eliminação da hanseníase, controle da hipertensão, controle da diabetes mellitus, ações de saúde bucal, ações de saúde da criança e ações de saúde da mulher ${ }^{(11)}$. No escopo da saúde da mulher é priorizada a assistência ao pré-natal, parto e puerpério, prevenção de câncer de colo do útero e de mama e planejamento familiar. O estabelecimento de prioridades e metas almeja a obtenção de melhores indicadores em diversos campos da saúde. Entretanto, ações no climatério não aparecem como uma prioridade explícita, mas como uma necessidade gerada segundo a demanda de cada região.

Assim, fica evidente nas falas dos profissionais e nas diretrizes da atenção básica que, apesar do discurso da integralidade ter sido adotado nas políticas públicas, no campo da saúde da mulher as ações ainda são organizadas tendo como referência central o período reprodutivo: as prioridades são planejamento sexual, reprodutivo e gestação. 


\section{As demandas das mulheres dos 45 aos 60 anos}

De acordo com os relatos dos/das profissionais, a procura pelo serviço de saúde pela mulher da faixa etária estabelecida neste estudo tem razões múltiplas que estão associadas a problemas físicos, psíquicos e sociais, tais como fogachos, depressão, ansiedade, irregularidade menstrual, dor de cabeça, pressão alta, sobrepeso, secura vaginal, falta de prazer sexual e irritabilidade, entre outras.

[...] elas reclamam muito dos fogachos, ressecamento, que não têm prazer sexual [...] (AE4)

[...] a saúde mental, depressão, agressão em casa, sobrepeso, deslipidemia... baixa autoestima [...] (N)

Quando a busca pelo atendimento não se justifica pelas queixas citadas, o profissional que a atende pode ou não abordar aspectos relativos ao climatério. Vejamos:

[...] elas vêm procurar outras coisas em relação à hipertensão, diabetes, papanicoloau, gestação... Durante a consulta, na anamnese entra esta questão da ultima menstruação, se já fez laqueadura, amenorréia etc. [...] (E1) [...] a gente não atende especificamente as mulheres no climatério, a gente atende as mulheres hipertensas, diabéticas que no final da consulta, quando a gente chega lá no exame físico inferior em relação à menstruação, ovário, essas coisas, a gente verifica se tem alguma alteração [...] (E2)

Um aspecto que nos chama atenção nos discursos dos profissionais é que parece haver o reconhecimento de demandas específicas das mulheres nessa faixa etária, mas não há ações dirigidas para esse grupo.

Teoricamente, na ESF as equipes de atenção básica são responsáveis por identificar usuários do serviço de saúde que apresentam fatores de risco para desenvolver certos agravos. Preconiza-se que as consultas estejam associadas à ações que promovam hábitos de vida saudáveis. No entanto, na USF estudada os relatos apontam para a priorização de ações protocolares, tais como o programa de detecção precoce dos cânceres de mama e colo de útero; de controle da hipertensão arterial e da diabetes mellitus. As ações de saúde parecem estar centradas nas doenças, nos distúrbios orgânicos, caracterizando a visão fragmentada do cuidado prevalente mesmo no âmbito da atenção básica. Nesse sentido, parece haver pouco espaço para a escuta de queixas/demandas das mulheres nessa faixa etária e, consequentemente, desenvolvimento de ações específicas $^{(3)}$.

Estudos apontam que a assistência à saúde da mulher na faixa etária dos 45 aos 60 anos deve ser organizada em várias etapas, qualificando a atenção com estratégias de cogestão $^{(4,11)}$. Nesse sentido, o acolhimento é o momento em que serão identificadas as queixas objetivas e subjetivas de cada mulher, tentando levantar os riscos e as vulnerabilidades para as doenças crônicas, alterações hormonais e câncer. A captação precoce dessas mulheres é muito importante no que se refere à prevenção dos agravos à saúde ${ }^{(4,11)}$. $O$ atendimento individual é feito por meio das consultas médicas e de enfermagem, cujo foco volta-se para a anamnese, exame físico e ginecológico, exames laboratoriais, aconselhamento nutricional e questões da vida pessoal da mulher que possam influenciar em sua saúde(12).

Vale lembrar que na abordagem da mulher no climatério é fundamental não se restringir ao aspecto biológico, pois o envelhecimento está cercado por aspectos psicológicos e culturais, além de mitos e desigualdades sociais e de gênero(13-14). Uma abordagem mais abrangente e focada nas necessidades específicas dos sujeitos pode ser uma motivação para a participação de ações de promoção da saúde.

\section{A organização das ações no cotidiano da USF}

Ao longo do processo de elaboração de políticas públicas de saúde no Brasil, nota-se a tendência de transição de modelos de organização que priorizam práticas centradas na doença e na assistência curativa para outros que buscam orientar-se ativamente em direção às práticas preventivas, de educação em saúde e à busca da qualidade de vida ${ }^{(15)}$. Nesta pesquisa pode-se perceber que o conceito de saúde adotado pelos profissionais ainda está muito associado aos aspectos curativos ou preventivos limitantes/limitados aos aspectos do processo saúde-doença.

Enquanto política pública nacional, a ESF integra uma rede de "ações" nas diversas frentes da atenção, ou seja, saúde da mulher, da criança e do adulto, entre outras. A atenção básica deve viabilizar a articulação dos diversos programas nos seus mais diversos aspectos, respeitando as particularidades de cada local/região do país, considerando a sua diversidade cultural, social, política e 
econômica. Vale salientar que, para além das evidências epidemiológicas que justificam a ênfase dada aos diversos programas de saúde, aspectos de maior complexidade devem ser considerados ${ }^{(15)}$.

No caso da unidade em estudo, queixas relacionadas à saúde mental foram citadas como sendo alterações que se refletem na qualidade de vida da mulher nessa faixa etária. Os profissionais disseram que, muitas vezes, a percepção dessas alterações se dá nas visitas domiciliares pelas ACS ou nas consultas, sem que a mulher faça por si mesma a associação de sintomas como tristeza, depressão, irritabilidade com as alterações hormonais:

[...] nas reuniões de equipe têm muitos casos de saúde mental... muitas mulheres que ficam em casa, sem fazer nada, sem contatos... problemas com os maridos [...] (F)

[...] sentem irritação... tem uma que achou que estava podre por dentro, pois sentia tantos problemas e era só menopausa $[. .$.$] (ACS3)$

[...] os casos que chegam pra mim são mais referentes à questão de saúde mental [...] $(P)$

De acordo com os relatos, na região onde a USF está localizada não há uma rede que dê conta das diversas demandas identificadas no cotidiano do serviço, tornando-se importante rever a organização da rede de assistência naquela área. A literatura indica que um dos principais limitantes das ações na ESF está nas dificuldades de implementação da integralidade; e no que se refere à saúde mental, muitos profissionais da ESF ainda não se sentem familiarizados com o universo do sofrimento psíquico(16).

Como especificado nas diretrizes do Ministério da Saúde, o acolhimento deve ocorrer no âmbito da atenção primária à saúde e a resolutividade dos problemas identificados deve vir na medida da sua identificação. No entanto, o que os dados desta pesquisa indicam é que a demanda por cuidados parece ser maior que a oferta de serviços, já que eles estão sempre comprometidos pelas dificuldades organizacionais, tais como número reduzido de equipes de saúde, dificuldade para manter um número de profissionais por um tempo suficiente para que seja estabelecido vínculo profissional-usuário e, consequentemente, reconhecer as necessidades individuais e coletivas. O discurso de um dos profissionais evidencia essa situação:
[...] as mulheres até vêm na consulta... aí o médico vai embora e passa um ano sem vir outro... às vezes aquela mulher que já marcou 3, 4 vezes e não consegue passar; ela até desiste... ela se acostuma com a situação e não vem mais $[\ldots](\mathrm{AE} 1)$

Outro aspecto importante identificado pela pesquisa é que, mesmo se tratando de uma unidade de saúde que utiliza ESF, a organização da assistência ainda segue a divisão entre saúde da mulher, saúde do adulto, saúde do idoso ou grupo de hipertensos, grupo de diabéticos, grupo de gestantes. Apesar de tratar-se de uma diretriz ministerial do programa de saúde da mulher, no discurso de alguns profissionais as mulheres nessa faixa etária, consideradas por muitos como estando no climatério, deveriam ter um programa à parte.

[...] os grupos de sala de espera são coisas esporádicas, não é específico para o climatério... é específico para os programas do ministério... as mulheres climatéricas sem sintomas entram no protocolo de saúde do adulto... então, se a mulher vem com uma queixa de climatério ela pode agendar com o generalista [...] (E2)

Essa organização do sistema que toma por base as especialidades de cada área acaba por fragmentar o serviço e torná-lo próximo ao ambiente hospitalar, comprometendo as premissas da atenção primária à saúde ${ }^{(17)}$. Esse modo de entender e, consequentemente, organizar a assistência no contexto da UBS/ESF, repercute na importância dada às ações de promoção da saúde ${ }^{(18)}$.

\section{Ações de promoção da saúde para as mulheres dos 45 aos 60 anos}

Ao serem questionados sobre as ações de promoção da saúde os profissionais falaram sobre a falta de adesão das mulheres às atividades, de um modo geral em todas as faixas etárias. Os motivos foram associados às características socioeconômicas, questões de gênero e a falta de profissionais. O baixo grau de instrução da população e falta de capacitação profissional para o trabalho em educação/promoção de saúde também foram apontadas: 
[...] elas vêm aqui (no bairro) só para dormir e vai (sic) trabalhar; normalmente elas deixam os filhos em creches $e$ saem para o emprego [...](E1)

[...] tá inserida no mercado de trabalho, tem que tomar conta da casa porque o homem não abre mão da posição machista... ela tem que cuidar dos filhos e isto dificulta a procura dela pelo serviço de saúde[...] (M)

[...] em questão de orientação do climatério não tem específico... nossa equipe é muito reduzida então já tem grupo de gestante, de hipertensão [...](AE1)

[...] falta instrução para as mulheres... falta direcionamento... nós temos dificuldades [...](AE1)

[...] nossos profissionais têm que se atualizar, têm que aperfeiçoar [...] (E2)

Considerando que as atividades de promoção da saúde configuram-se como uma prática essencial na atenção primária é importante investir na elaboração de metodologias e estratégias para essas ações. Estudos demonstram que, no período de transição entre a fase reprodutiva e a fase não reprodutiva, atividades educativas que busquem esclarecer sobre as mudanças dessa nova fase da vida ajudam a mulher a vivenciar os sintomas e sentimentos classificados como sendo do climatério $^{(14,17)}$. Ao longo da pesquisa ficou evidente que os profissionais de saúde fazem associação das queixas das mulheres nessa faixa etária com o conjunto de sinais e sintomas típicos do climatério. E parece haver esforços no sentido de minimizar/aliviar esses sintomas:

[...] a gente conta com isso na rede, né? Mas só tem que atender a todos os requisitos do protocolo pra gente entrar com THR (Terapia de Reposição Hormonal) com segurança máxima... Eu costumo usar também produtos naturais, tipo isoflavona, tibolona, então... e o resultado é bastante razoável. Uma coisa que eu procuro estimular muito é exercício físico $[. .].(M)$

Nesse discurso fica evidente que há uma preocupação com a segurança no uso de medicamentos e uma orientação no sentido de auxiliar as usuárias a desenvolverem estratégias dirigidas a uma vida saudável. A USF, muitas vezes, carece de um sistema de referência e contrarreferência eficaz/efetivo. O fato de não existir médicos especialistas na unidade, considerada porta de entrada da rede de atenção básica, pode dificultar o acesso das mulheres às ações propostas. O relato a seguir sugere a necessidade de deslocamentos, o que pode implicar descontinuidade do acompanhamento:

[...] nós não temos medicamentos para TRH... elas são encaminhadas para o ginecologista [...] (E1)

[...] no momento não tem TRH na nossa unidade... através dos exames, se ele (o médico) ver alguma alteração ou ele pede um ultrassom ou ele encaminha para o ginecologista $[\ldots](\mathrm{E} 2)$

Nesse contexto, a organização da atenção básica à saúde (ABS) deve ser pautada pela integração dos serviços. O atendimento e o acesso aos serviços especializados devem ser estruturados com a efetivação de uma porta de entrada preferencial com poder de resolutividade, dispondo, por exemplo, de uma lista ampliada de medicamentos e maior acesso a exames complementares. A existência de um serviço de primeiro contato facilita a formação de vínculos e a coordenação dos cuidados e, assim, o sistema se retroalimenta ${ }^{(18)}$.

Tendo os serviços de ABS como porta de entrada, muitas vezes a captação dos usuários de saúde se dá no momento da procura por um cuidado específico. O Papanicolau, por exemplo, acaba sendo uma porta de entrada para os serviços de saúde para as mulheres nessa faixa etária, pois através do exame elas são orientadas a passar com o médico para realizar outros de rotina, mesmo não apresentando sintomas. Segundo a fala de um dos médicos da unidade, nas consultas das mulheres dos 45-60 anos o foco está na prevenção dos cânceres de mama e útero:

[...] no PSF a gente tem muita coisa pra focar... eu procuro passar isso pra minha equipe... duas preocupações... as alterações que acontecem... nessa fase e o câncer... primeiro eu prezo pelo "papa", em segundo lugar, pela mamografia e depois pela USG (ultrassonografia) transvaginal [...] (M)

Essa é uma preocupação consonante com os estudos e pesquisas sobre o câncer de colo de útero e câncer de mama ${ }^{(19-20)}$. Já que esses indicam que, quando ações de atenção primária dirigidas a esta população funcionam, as ações de rastreamento citadas pelo médico permitem que essas patologias possam ser identificadas no início e as chances de cura tornarem-se muito altas.

Quando questionados sobre a principal dificuldade na assistência às mulheres nessa faixa etária as respostas 
não divergiram muito entre os profissionais de saúde, apontando a falta de tempo e de informação das mulheres sobre as questões de saúde como principal fator que distancia esses sujeitos da unidade. Assim, outra estratégia utilizada para ampliar o acesso é a organização de campanhas de coleta de exames, nas quais a unidade de saúde amplia seu horário de funcionamento:

\section{[...] a gente está aberto no sábado pra fazer o Papanicolau} nas mulheres que não têm condições de vir durante a semana, E nós temos programado desde o começo do ano mais duas campanhas pra poder pegar esse pessoal que tem dificuldade por causa do horário [...] (E2)

Fica evidente nessa fala que há esforços para realizar campanhas e garantir uma ampliação das ações de promoção da saúde. No entanto, essas iniciativas buscam ampliar a cobertura dos cuidados para todas as mulheres e não necessariamente têm foco nas mulheres dos 45 aos 60 anos. De qualquer forma, fica explícito também o reconhecimento da necessidade de modificação dos horários de atendimento na unidade para possibilitar o acesso das mulheres trabalhadoras ao serviço. É necessário pensar novos modos de organizar os serviços de saúde a fim de garantir que todas as mulheres tenham acesso a eles.

\section{Agente comunitário como facilitador}

Os agentes comunitários de saúde são elementoschave para a implementação das ações de saúde, já que conhecem a dinâmica social, os valores, as formas de organização e os repertórios linguísticos que circulam entre os moradores. Assim tornam-se facilitadores para a equipe, tanto como mediadores entre o conhecimento popular e o conhecimento tecnocientífico, quanto na identificação de demandas da população local(21).

Corroborando com essa proposição, os profissionais entrevistados destacaram o papel do ACS na assistência às mulheres:

[...] elas (as mulheres nessa faixa etária) acabam aparecendo através das agentes comunitárias [...] (N)

[...] quando ela têm alguma dúvida, ela procura a agente comunitária, a agente comunitária orienta a vir na unidade $[\ldots](\mathrm{AE} 4)$
Essas falas evidenciam que os ACS de fato são considerados como facilitadores das relações entre a população e a equipe de saúde.

Muitas vezes são as próprias ACS que dão orientações sobre saúde da mulher durante as visitas domiciliares, ficando responsáveis pela verificação da frequência com que elas realizam os exames preventivos. Como indica o relato abaixo:

[...] Então a gente tem os agentes comunitários... durante as suas visitas eles têm um formulário que eles preenchem da mulher em relação à pergunta "quando foi o seu ultimo Papanicolau? Você fez Papanicolau esse ano?". E muitas vezes eles orientam [...] (E2)

Importante destacar que a visita domiciliar é a principal atividade do ACS e que este também seria um momento para desenvolver atividades de promoção de saúde. Porém, como elas/eles assumem muitas atribuições, nem sempre é possível sistematizar as ações dirigidas à identificação das demandas das mulheres nessa faixa etária. A sobrecarga de trabalho é citada como um dificultador dessas ações:

[...] Nós não temos pernas pra ir buscar porque a gente tem uma meta de consulta, uma meta de grupo, meta disso, meta daquilo. Então o agente comunitário é o nosso portavoz, né? Eles sabem das queixas dos clientes e, normalmente, todas as equipes se reúnem durante uma hora, então eles expõem os casos [...] (E2)

A sobrecarga de trabalho das equipes e o modo como a ESF está organizada na região, dificulta a ampliação das ações para além das consultas no interior das unidades e aquelas previstas para as visitas domiciliares. Essa situação configura-se como um dos paradoxos da ESF, no qual se espera que sejam realizadas ações de promoção da saúde; porém, a organização do trabalho é focada no número de indivíduos e de famílias atendidas. Tal constatação conduz necessariamente à reflexão sobre quanto o modelo neoliberal de atenção à saúde ainda é dominante ${ }^{(22)}$.

Na fala dos ACS percebe-se a facilidade delas em abordar essas mulheres e conversar sobre diversos assuntos, graças ao vínculo que elas estabeleceram com a comunidade: 
[...] Ah, a agente já tem um vínculo com a familia, né? Então ela tem uma certa confiança de uma pra outra; ai elas acabam se abrindo, conversando. Então, assim, não tem muita dificuldade, porque, assim, a gente já tem essa experiência [...] (ACS 2)

[...] Eu não tenho dificuldades pra abordar, tanto é que sou esclarecida... a gente conhece, a gente conversa bastante com elas [...] (ACS 5)

As visitas domiciliárias feitas pelas agentes de saúde configuram-se uma intervenção social com grande potencial de transformação. $O$ uso da linguagem e o momento apropriados, bem como o interesse compartilhado pelo tema em exposição, são atributos que podem garantir o alcance dos objetivos da educação em saúde e devem ser considerados na elaboração dos projetos para grupos de educação em saúde (21).

\section{CONSIDERAÇÕES FINAIS}

Os resultados da pesquisa nos permitem concluir que na unidade pesquisada não existem ações de saúde específicas para as mulheres da faixa etária dos 45 aos 60 anos, e estas são atendidas somente quando procuram o serviço espontaneamente. 0 foco das ações programáticas implementadas estão nas prioridades estabelecidas pela ESF - os hipertensos, os diabéticos, as gestantes, as crianças e os idosos.

\section{REFERÊNCIAS}

1. Freitas GL, Vasconcelos CTM, Moura ERF, Pinheiro AKB. Discutindo a política de atenção à saúde da mulher no contexto da promoção da saúde. Rev. Eletr. Enf. [Internet]. 2009;11(2):424-8. Available from:

http://www.fen.ufg.br/revista/v11/n2/v11n2a26.htm

2. De Lorenzi DRS, Catalani LB, Moreira K, Ártico GR. Assistência à mulher climatérica: novos paradigmas. Rev. Bras. Enferm. Brasília. [Internet] 2009[cited 2012 fev 15];62(2):287-93. Available from:

http://www.scielo.br/pdf/reben/v62n2/a19v62n2.pdf 3. Pereira QLC, Siqueira HCH. O olhar dos responsáveis pela política de saúde da mulher climatérica; Esc Anna Nery Rev Enferm. [Internet] 2009[cited 2012 fev 15]; 13(2): 366-71. Available from:

http://www.scielo.br/pdf/ean/v13n2/v13n2a18.pdf 4. Secretaria de Atenção à Saúde, Ministério da Saúde. Departamento de Ações Programáticas Estratégicas. Manual de atenção à mulher no climatério/menopausa. Brasília (Brasil): Ministério da Saúde, 2008. 192p.

5. Mori ME, Coelho VLD, Estrella RCN. Sistema Único de Saúde e políticas públicas: atendimento psicológico à mulher na menopausa no Distrito Federal, Brasil. Cad. Saúde Pública, Rio de Janeiro. [Internet] 2006[cited 2012 fev 15]; 22(9):1825-33. Available from: http://www.scielo.br/pdf/\%0D/csp/v22n9/06.pdf
O estudo demonstra também que os/as profissionais de saúde que atuam na ESF têm consciência da importância de desenvolver ações específicas de atenção básica para as mulheres nessa faixa etária. Porém, uma multiplicidade de fatores contribui para que essas ações não sejam priorizadas, como a falta de tempo; a necessidade de atender as prioridades estabelecidas pela ESF; o modo como as ações de promoção e educação em saúde está organizado.

Assim, apesar de focalizar apenas uma unidade de saúde da família, o estudo contribui para uma reflexão sobre a inexistência de ações de promoção de saúde específicas para mulheres na faixa etária dos 45 aos 60 anos, conforme preconizado pelas diretrizes da Política Nacional de Atenção Integral à Saúde da Mulher. Os resultados apontam para a necessidade de criar estratégias e programas que incluam ações de atenção primária para essa população.

Finalmente, considera-se que para além da criação de políticas públicas de saúde e seus diversos programas, faz-se necessário articulá-los e buscar garantir que as diretrizes sejam implementadas e concretizadas no cotidiano dos serviços. Também é imprescindível que a diversidade da população seja considerada nas ações de atenção primária à saúde.

6. Ministério da Saúde. Política Nacional de Atenção Integral a Saúde da Mulher: Princípios e diretrizes. Brasília (Brasil): Ministério da Saúde, 2004. 82p.

7. Secretaria Municipal de Saúde de São Paulo. Lei Municipal No 13.536/2003. (Brasil). Cria o Programa de Atendimento Integral e Humanizado às Mulheres em Estado de Climatério ou Pósclimatério. São Paulo (Brasil). Available from:

http://www.prefeitura.sp.gov.br/cidade/secretarias/saude/legis lacao/index $\cdot$ php? $\mathrm{p}=6360$.

8. Conill EM. Políticas de atenção primária e reformas sanitárias: discutindo a avaliação a partir da análise do Programa Saúde da Família em Florianópolis, Santa Catarina, Brasil, 1994-2000. Cad. Saúde Pública [Internet] 2002 [cited 2013 Jun 08];18

Suppl.:191-202. Available from:

http://dx.doi.org/10.1590/S0102-311X2002000700019.

9. Berni NIO, Luz MH, Kohlrausch SC. Conhecimento, percepções e assistência à saúde da mulher no climatério; Rev. Bras.

Enferm., Brasília. [Internet] 2007 [cited 2012 fev 15];60(3):299306. Available from:

http://www.scielo.br/pdf/reben/v60n3/a10.pdf

10. Valença CN, Germano RM. Concepções de mulheres sobre menopausa e climatério. Rev. Rene. 2010;11(1):161-71.

11. Ministério da Saúde. Regionalização da Assistência a Saúde: Aprofundando a descentralização com equidade no acesso: Norma Operacional da Assistência a Saúde: NOAS-SUS 01/02 e 
Portaria MS/GM n³73/02. Brasília (Brasil): Ed. Revista e atualizada, 2002.116p.

12. Diógenes MAR, Linard AG, Teixeira CAB. Comunicação, acolhimento e educação em saúde na consulta de enfermagem em ginecologia. Rev. Rene. Fortaleza. Out./dez.2010; 11(4):3846.

13. Mendonça EAP. Representações médicas e de gênero na promoção da saúde no climatério/menopausa. Ciência \& Saúde Coletiva. 2004;9(3):751-62.

14. Oliveira DM, Jesus MCP, Merighi MAB. O climatério sob a ótica de mulheres assistidas em uma unidade de saúde da família de juiz de fora -minas gerais. Rev. APS. [Internet] 2008 [cited 2012 mar 25];11(1):42-53. Available from: http://www.ufjf.br/nates/files/2009/12/042-053.pdf. 15. Campos, CEA. O desafio da integralidade segunda as perspectivas da vigilância da saúde e da saúde da família. Ciência \& Saúde Coletiva [Internet] 2003[cited $2012 \mathrm{fev}$ 15];8(2):569-84. Available from: http://www.scielosp.org/pdf/csc/v8n2/a18v08n2.pdf 16. Delfini PSS, Sato MT, Antoneli PP, Guimaraes POS. Parceria entre CAPS e PSF: o desafio da construção de um novo saber. Ciênc. saúde coletiva, Rio de Janeiro. [Internet] 2011[cited 2012 fev 15];14 (1): 1483-92. Available from: http://www.scielosp.org/pdf/csc/v14s1/a21v14s1.pdf 17. Silva, ASR. Assistência realizada por enfermeiros do PSF a mulher no climatério, Caderno de cultura e ciência, Ano IV. [Internet] 2009[cited $2012 \mathrm{fev} 15]$; 1(1):29-38. Available from: http://periodicos.urca.br/ojs/index.php/cadernos/article/viewFil e/169/111

18. Escorel S, Giovanella L, Mendonça MHM, Senna MCM. O programa de Saúde da Família e a construção de um novo modelo para a atenção básica no Brasil. Rev Panam Salud Publica/Pan Am J Public Health. [Internet] 2007[cited $2012 \mathrm{fev}$ 15]; 21(2): 164-176. Available from:

http://www.scielosp.org/pdf/rpsp/v21n2-3/11.pdf

19. Bim CR, Pelloso SM, Carvalho MDB, Previdelli ITS.

Diagnóstico precoce do câncer de mama e colo uterino em mulheres do município de Guarapuava, Paraná, Brasil. Rev. esc. enferm. USP [Internet] 2010 [cited 2013 June 08]; 44(4): 940946. Available from: http://dx.doi.org/10.1590/50080$\underline{62342010000400012}$

20. INCA (Instituto Nacional de Câncer) José Alencar Gomes da Silva. Coordenação Geral de Ações Estratégicas. Coordenação de Prevenção e Vigilância.Estimativa 2012: incidência de câncer no Brasil. Rio de Janeiro: INCA, 2011.118 p. Available from: http://www.inca.gov.br/estimativa/2012/index.asp?id=5 21. Pinto AAM, Fracolli LA. O trabalho do agente comunitário de saúde na perspectiva da promoção da saúde: considerações práxicas. Rev. Eletr. Enf. [Internet]. 2010;12(4):766-9. Available from: http://www.fen.ufg.br/revista/v12/n4/v12n4a24.htm. 22. Brigagão JIM, Gonçalves R. Oficinas de promoção de saúde: discutindo os dilemas do cotidiano de um grupo de agentes comunitárias de saúde. Paidéia [Internet] 2009[cited 2012 fev 15];19(44):387-393. Available from: http://www.scielo.br/pdf/paideia/v19n44/a12v19n44.pdf

Artigo recebido em 14/05/2012.

Aprovado para publicação em 23/05/2013.

Artigo publicado em 30/09/2013. 